\title{
A Facile Microwave-Assisted Synthesis of Some Fused Pyrimidine Derivatives
}

\author{
S. A. AL-ISSA \\ Department of Chemistry, Faculty of Science, \\ Princess Nora Bint Abdul Rahman University, Riyadh, Saudi Arabia. \\ ${ }^{*}$ Corresponding author E-mail: Seham.alissa@gmail.com \\ http://dx.doi.org/10.13005/ojc/300209
}

(Received: March 05, 2014; Accepted: March 31, 2014)

\begin{abstract}
The highly accelerated synthesis of thienopyrimidinones, theino- pyrimidines, thioxotheinopyrimidinones and a thienotriazolopyrimidinone derivatives under microwave irradiation is reported. Compared to conventional conditions, microwaves method offered several advantage likes short time, good yields, simple procedure, mild conditions and easy workup. The structure of synthesized compounds have been characterized on the basis of their elemental analysis and spectral data, and screened for their antimicrobial activity.
\end{abstract}

Key words: Thienopyrimidine, Thioxothienopyrimidine, Triazolopyrimidinone, Microwave Irradiation.

\section{INTRODUCTION}

Pyrimidines and fused pyrimidines compounds, are a big family of the heterocyclic compounds, which have been focus of great interest because of their wide range of biological and pharmacological activities as antibacterial and antifungal activities ${ }^{1-4}$ anticancer ${ }^{5-8}$, antiinflammatory ${ }^{9}$ and many thieno[2,3-d] pyrimidine derivatives have been proved to use in case of Alzheimer and Parkinson disease ${ }^{10}$.

Microwave irradiation has attracted attention of organic synthesis due to the reduction in reaction times, good yields and cleaner reaction than conventional procedure ${ }^{11-16}$. There are several examples describing the advantage of using microwave irradiation for synthesis the thieno [2,3d]pyridine-4 $(3 \mathrm{H})$ ones for example, Hesse reported the synthesis of thieno[2,3-d]pyrimidin$4(3 \mathrm{H})$-ones under microwave irradiation in good yield ${ }^{17}$.

It seemed most interesting to extend the scope of synthesize of pyrimidine derivatives having active moieties under microwave irradiation with the aim to evaluate their biological activity as antibacterial or antifungal agents. 


\section{EXPERIMENTAL}

Melting point are uncorrected. Microwave reaction were performed on a microwave oven start SYNTH-Milestone-open vessel. IR spectra were recorded in potassium bromide on Perkin-Elmer 380 and 387 spectrometer. ${ }^{1} \mathrm{H}-\mathrm{NMR}$ spectra were run on a JEOL-JNMGX-300 and Junm Ex-400 in DMSO- $\mathrm{d}_{6}$ or $\mathrm{CDCl}_{3}$ using TMS as internal standard and chemical shift were expressed as part of million, ppm ( values) against TMS and internal references. Electron impact (E1) MS spectra were obtained with aid of a ShimaDZU Gemsqp 5050. A spectrometers at $70 \mathrm{ev}$. Elemental analysis were recorded on a Heruss $\mathrm{CHN}$ analyser.

General procedure for synthesis of 5,6disubtituted thieno[2,3-d]pyrimidin-4(3H)-ones $\left(4_{\mathrm{a}-\mathrm{c}}\right)$

A mixture of $1_{a, b}(1 \mathrm{mmol})$ and formamide (5-6 mmol) in ( $1 \mathrm{mmol}$ ) acetic acid, was irradiation in the microwave oven for $10 \mathrm{~min}$. at $(300 \mathrm{~W}) 130^{\circ} \mathrm{C}$. The reaction mixture was cooled and poured into iced-cold water, filtered, washed and recrystallized from ethanol.

5,6-Dimethyl-3H-thieno[2,3-d]pyrimidin-4-one (4 M.p. $180-183^{\circ} \mathrm{C}$; IR $\left(\mathrm{cm}^{-1}\right)$ : 3320,$1670 ;{ }^{1} \mathrm{H}-$ $\operatorname{NMR}\left(\mathrm{DMSO}-\mathrm{d}_{6}\right)$ 1.7(s, 3H, CH3), 2.0(s, 3H, CH3), $7.80(1 \mathrm{H}, \mathrm{s}$, pyrimidine $-\mathrm{H}), 10.00$ (br. s, $1 \mathrm{H}, \mathrm{NH})$; MS: m/z (\%), 180[M+] (95) $\left(\mathrm{C}_{8} \mathrm{H}_{8} \mathrm{~N}_{2} \mathrm{OS}\right) ; \mathrm{CHN}$ anal. calcd \%: C:53.33; H: 4.44; N:15.55; Found: C: 53.45; $\mathrm{H}: 4.30 ; \mathrm{N}: 15.56$.

\section{5,6,7,8-Tetrahydro-3H-benzo[4,5]thieno[2,3- d]pyrimidin-4-one $\left(4_{b}\right)$}

M.p. $257-259^{\circ} \mathrm{C}, \mathrm{IR}\left(\mathrm{cm}^{-1}\right)$ : br. 3400, 1670; ${ }^{1} \mathrm{H}-\mathrm{NMR}\left(\mathrm{DMSO}-\mathrm{d}_{6}\right): 1.8-1.87\left(\mathrm{~m}, 4 \mathrm{H}, \mathrm{CH}_{2}\right.$ at C-6 and $\mathrm{C}-7), 2.65-2.70\left(\mathrm{~m}, 2 \mathrm{H}, \mathrm{CH}_{2}\right.$ at $\left.\mathrm{C}-5\right) ; 2.80-2.89$ $\left(\mathrm{m}, 2 \mathrm{H}, \mathrm{CH}_{2}\right.$ at $\left.\mathrm{C}-8\right), 7.80(\mathrm{~s}, 1 \mathrm{H}$, pyrimidine $-\mathrm{H})$, 10.90 (br. s, $1 \mathrm{H}, \mathrm{NH})$; MS: m/z (\%) $206\left[\mathrm{M}^{+}\right](90)$ $\left(\mathrm{C}_{10} \mathrm{H}_{10} \mathrm{~N}_{2} \mathrm{OS}\right)$; CHN Anal. Calcd . \% : C, 58.25; $\mathrm{H}, 4.85$; N, 13.59 Found: C, 58.30; H, 4.83; N, 13.60 .

\section{3,5,6,7,8,9-Hexahydrocyclohepta[4,5]thieno[2,3-} d]pyrimidin-4-one ( $\left.4_{c}\right)$

M.p.: $212-215^{\circ} \mathrm{C}$; IR $\left(\mathrm{cm}^{-1}\right)$ : br 3410, 1710; ${ }^{1} \mathrm{H}-\mathrm{NMR}\left(\mathrm{DMSO}-\mathrm{d}_{6}\right)$ : $1.56-1.58\left(\mathrm{~m}, 4 \mathrm{H}, 2 \mathrm{CH}_{2}\right.$ at $\mathrm{C}-6$ d C-7); $1.83-189\left(\mathrm{~m}, 2 \mathrm{H}, \mathrm{CH}_{2}\right.$ at C-8), 2.70-283 (m, $2 \mathrm{H}, \mathrm{CH}_{2}$ at C-5), 2.96-3.0 (m, $2 \mathrm{H}, \mathrm{CH}_{2}$ at C-9), 8.01 (s, $1 \mathrm{H}$, pyrimidine $-\mathrm{H}$ ) 12.5( br. s, $1 \mathrm{H}, \mathrm{NH}) ; \mathrm{MS}: \mathrm{m} / \mathrm{z}$ (\%) $220\left[\mathrm{M}^{+}\right]$(100) $\left(\mathrm{C}_{11} \mathrm{H}_{12} \mathrm{~N}_{2} \mathrm{OS}\right)$; CHN . Anal. Calcd. \%: C:60.00; H:5.45, N: 12.72; Found: C: $60.10, \mathrm{H}$ : $5.47 ; \mathrm{N}: 12.70$.

\section{4-Chloro-5,6,7,8-tetrahydrobenzo[4,5] thieno[2,3-} d] pyrimidine (7):

\section{Conventional method}

A mixture of $4_{b}(5 \mathrm{mmol})$ and phosphorus oxychloride $(15 \mathrm{ml})$ was heated under reflux for 20 h. After completion of reaction, The excess phosphorus oxychloride was removed by distillation under reduced pressure ,the residue left was titrated with sodium bicarbonate solution (10\%); the solid separated was filtered, dried and recrystallized from toluene.<smiles>[X]C(=O)c1c(N)sc([R])c1[R]</smiles>

Scheme 1: 


\section{Microwave method}

A mixture of $4_{b}(5 \mathrm{mmol})$ and phosphorus oxychloride $(15 \mathrm{ml})$ was irradiated in microwave oven at $(60 \mathrm{~W}) 120^{\circ} \mathrm{C}$ for $15 \mathrm{~min}$.. After completion of reaction, The workup was carried out as described for conventional method. M.p: $93-96^{\circ} \mathrm{C}$; IR $\left(\mathrm{cm}^{-1}\right): 1620,1580 ;{ }^{1} \mathrm{H}-\mathrm{NMR}\left(\mathrm{DMSO}_{-} \mathrm{d}_{6}\right): 1.90-$ 1.94, $\left(\mathrm{m}, 4 \mathrm{H}, 2 \mathrm{CH}_{2}\right.$, at $\mathrm{C} 6$ \& $\left.\mathrm{C} 7\right), 2.90-2.94(\mathrm{~m}, 2 \mathrm{H}$, $\left.\mathrm{CH}_{2}, \mathrm{C}-5\right), 3.00-3.40\left(\mathrm{~m}, 2 \mathrm{H}, \mathrm{CH}_{2}\right.$ at C-8), $8.72(\mathrm{~d}$, $1 \mathrm{H}$ pyrimidine $-\mathrm{H})$; MS: $\mathrm{m} / \mathrm{z}(\%) 224\left[\mathrm{M}^{+}\right]$(56\%) ( $\left.\mathrm{C}_{10} \mathrm{H}_{9}{ }^{35} \mathrm{ClN} \mathrm{N}_{2} \mathrm{~S}\right), 226[\mathrm{M}+2](30)\left(\mathrm{C}_{10} \mathrm{H}_{9}{ }^{37} \mathrm{CIN}_{2} \mathrm{~S}\right) ; \mathrm{CHN}$ : Anal. Calcd.: C: 53. 57: H: 4.01; N: 12.50.Found C: 53.21, H: 4.20, N: 12.49 .
General procedure for synthesis of $4-\mathrm{N}$-(aryl)5,6,7,8-tetrahydrobenzo [4,5]thieno[2,3-d] pyrimidines (8):

conventional method

A solution of compound $7(10 \mathrm{mmol})$ and aryl amine $(2.0 \mathrm{mmol})$ in 2-propanol $(30 \mathrm{~mL})$ was stirred under reflux for $12 \mathrm{~h}$., the solvent was removed under reduced pressure and the solid product was filtered dried and washed several times with petroleum ether.

Microwave Method:

A mixture of compound $7(1.0 \mathrm{mmol})$ and aryl amine $(2.0 \mathrm{mmol})$ in 2-propanol $(10 \mathrm{~mL})$ was stirred for $5 \mathrm{~min}$., then the mixture was irradiated in<smiles>[X]C(=O)c1c(N)sc([R2])c1Br</smiles><smiles>CC[CH-]CN</smiles><smiles>[R]c1sc2c(c1[R])C([X])(CO)CN2C(C)N</smiles><smiles>[R]c1sc2ncnc(O)c2c1[R]</smiles>

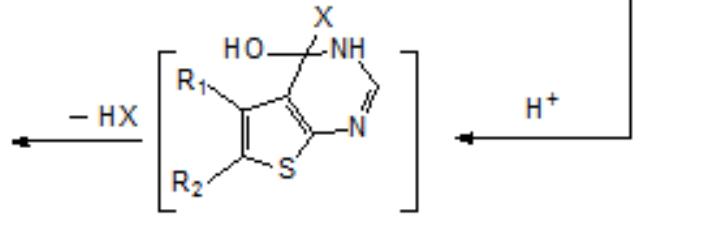

Scheme 2:<smiles>OC1NC=NC2SC3=C(CCCC3)C12</smiles>

5<smiles>Oc1ncnc2c1C1CCCCC1S2</smiles>
MW method $(60 \mathrm{~W}) 120$ aC, $15 \mathrm{~min}$
Conven. metrod $-\mathrm{ref} 20 \mathrm{~h}$.

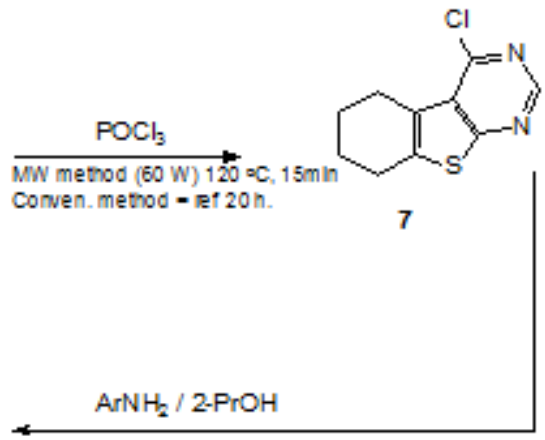

MN metrod ( $60 \mathrm{~W}$ ) $100^{\circ} \mathrm{C}, 20 \mathrm{~min}$ Conven, method: ret $12 \mathrm{~h}$.

Compound No. $\mathrm{R}_{1}, \mathrm{R}_{2} \quad \mathrm{Ar}$

$8_{\mathrm{a}} \quad\left(\mathrm{CH}_{2}\right)_{4} \quad 4-\mathrm{CH}_{3}$

$8_{b}^{a} \quad\left(\mathrm{CH}_{2}\right)_{4} \quad 3-\mathrm{CF}_{3}$

8 c $\quad\left(\mathrm{CH}_{2}\right)_{4}$ 2,5-di $\mathrm{CH}_{3}$

Scheme 3: 
the microwave oven at $(60 \mathrm{~W}) 100^{\circ} \mathrm{C}$ for $20 \mathrm{~min}$. The workup was carried out as described for conventional method.

\section{4-N-(4-methylphenyl)-5,6,7,8-tetrahydro[4,5]} thieno[2,3-d] pyrimidine $\left(\mathbf{8}_{\mathrm{a}}\right)$

M.p . ${ }^{\circ} \mathrm{C}: 235-237^{\circ} \mathrm{C}$; IR $\left(\mathrm{cm}^{-1}\right)$ : br. 3410 ; 1620; ${ }^{1} \mathrm{H}-\mathrm{NMR}\left(\mathrm{DMSO}-\mathrm{d}_{6}\right): 1.85-1.98\left(\mathrm{~m}, 4 \mathrm{H}, \mathrm{CH}_{2}\right.$ at C-6, C-7) 2.28(s, 3H, $\left.\mathrm{CH}_{3}\right), 2.34-2.64\left(\mathrm{~m}, 2 \mathrm{H}, \mathrm{CH}_{2}\right.$ at C-5) 2.9-3.04 (m, 2H, $\mathrm{CH}_{2}$ at C-8), 7.1-7.80 (m,4H,ArH) , 7.95-8.1, ( br.s, $1 \mathrm{H}, \mathrm{NH}), 8.22(\mathrm{~s}, 1 \mathrm{H}$, pyrimidinH). MS: $\mathrm{m} / \mathrm{z}(\%) 295\left[\mathrm{M}^{+}\right](95 \%) .\left(\mathrm{C}_{17} \mathrm{H}_{17} \mathrm{~N}_{3} \mathrm{~S}\right)$; $\mathrm{CHN}$ : Anal. Calcd $\quad$. C:69.15.H: 5.76; N: 14.23. Found: C: $69.30, \mathrm{H}: 5.31$; N: 14.34 .

4-N-(3-Trifluoromethylphenyl)-5, 6, 7,8tetrahydrobenzo[4,5]thieno [2,3-d] pyrimidine $\left(\mathbf{8}_{\mathrm{b}}\right)$ M.p ${ }^{\circ}$ C. 224-225 ${ }^{\circ} \mathrm{C}$; IR $\left(\mathrm{cm}^{-1}\right)$ : br. 3390, 1610;
${ }^{1} \mathrm{H}-\mathrm{NMR}$ (DMSO- $\mathrm{d}_{6}$ ): $1.80-1.87\left(\mathrm{~m}, 4 \mathrm{H}, 2 \mathrm{CH}_{2}\right.$ at C-6 d C-7), 2.40-2.60(m, $2 \mathrm{H}, \mathrm{CH}_{2}$ at C-5), 2.93-3.00 (m, $2 \mathrm{H}, \mathrm{CH}_{2}$ at $\left.\mathrm{C}-8\right), 7.31-7.80(\mathrm{~m}, 4 \mathrm{H}, \mathrm{Ar}-\mathrm{H}), 8.10(\mathrm{~s}$, $1 \mathrm{H}$, pyrimidin-H) ,8.7-8.9 (br. s, $1 \mathrm{H}, \mathrm{NH}) ; \mathrm{MS}: \mathrm{m} / \mathrm{z}$ (\%) $349(100)\left(\mathrm{C}_{17} \mathrm{H}_{14} \mathrm{~F}_{3} \mathrm{~N}_{3} \mathrm{~S}\right)$; $\mathrm{CHN}$ : Anal. Calcd. (\%) C: $58.45 ; \mathrm{H}: 4.01$; N: 12.03; Found: C, 58.36; H: 4.36; $\mathrm{N}: 12.98$.

\section{4-N-(2,5-Dimethylphenyl)-5,6,7,8-[4,5]thieno[2,3- d] pyrimidine $\left(\mathbf{8}_{\mathrm{c}}\right)$}

M.p ${ }^{\circ} \mathrm{C} .213-215^{\circ} \mathrm{C} ; \mathrm{IR}\left(\mathrm{cm}^{-1}\right)$ : br. 3140, 1610; ${ }^{1} \mathrm{H}-\mathrm{NMR}\left(\mathrm{DMSO}-\mathrm{d}_{6}\right): 1.80-1.87\left(\mathrm{~m}, 4 \mathrm{H}, 2 \mathrm{CH}_{2}\right.$ at C-6 d C-7), 2.31(s, 3H, $\left.\mathrm{CH}_{3}\right), 2.40\left(\mathrm{~s}, 3 \mathrm{H}, \mathrm{CH}_{3}\right), 2.44(\mathrm{~m}$, $2 \mathrm{H}, \mathrm{CH}_{2}$ at $\left.\mathrm{C}-5\right)$, 2.94-2.97 (m, $2 \mathrm{H}, \mathrm{CH}_{2}$ at $\left.\mathrm{C}-8\right)$, 7.16-7.80 (m, 3H, Ar-H), $8.18(\mathrm{~s}, 1 \mathrm{H}$, pyrimidine $-\mathrm{H})$ ,8.41( br. s, $1 \mathrm{H}, \mathrm{NH})$; MS: m/z (\%); $309(60 \%)$. $\mathrm{C}_{18} \mathrm{H}_{19} \mathrm{~N}_{3} \mathrm{~S}$; $\mathrm{CHN}$ :Anal. Calcd. (\%): C, 69.90, H: 6.14, $\mathrm{N}: 13.59$; Found: C: 69.93; H 6.92.

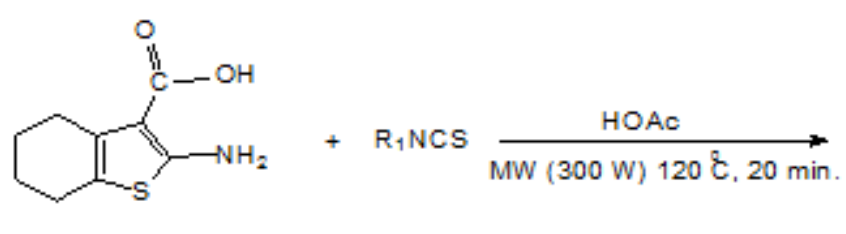

$1 \mathrm{~b}$<smiles></smiles>

10<smiles>[R]N1c2sc3c(c2[C@H](O)N([R1])C1S)CCCC3</smiles>

$11 \mathrm{a}-\mathrm{c}$

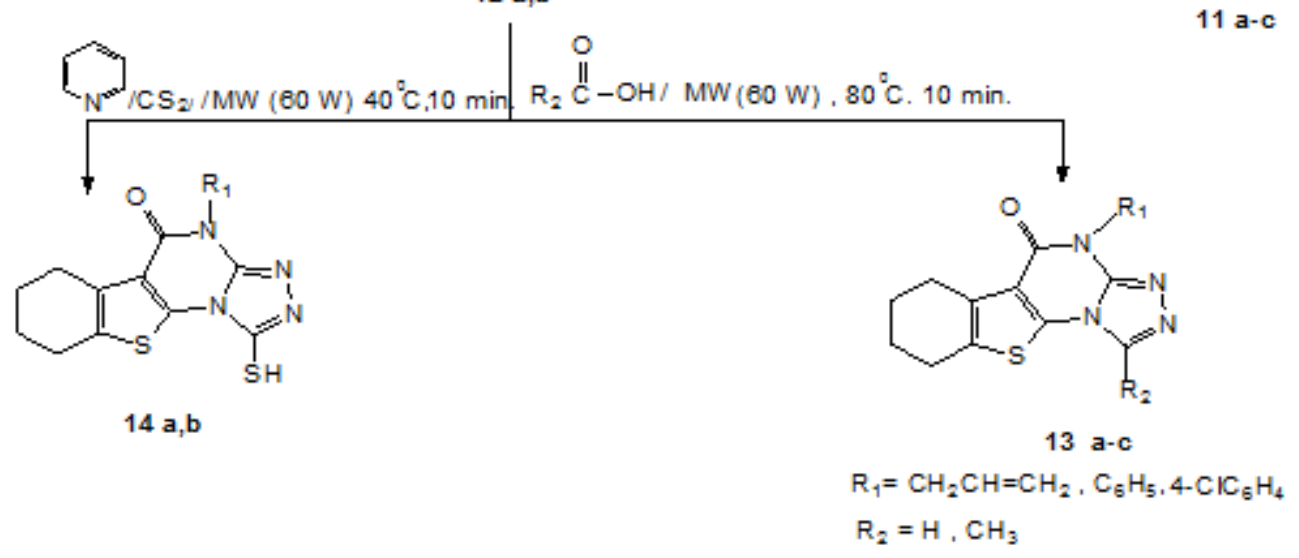

Scheme 4: Synthetic route of compounds 11-14 
General procedure for synthesis of 3,5,6trisubtituted-1H-2-thioxo thieno[2,3,-d] pyrimidin4-ones(11)

A mixture of $1_{\mathrm{b}}(1 \mathrm{mmol})$ and the appropriate isothiocyanate $(1.1 \mathrm{mmol})$ in $(10 \mathrm{ml})$ acetic acid, the mixture was irradiated in microwave oven at $(300 \mathrm{~W}) 120^{\circ} \mathrm{C}$ for $20 \mathrm{~min}$. The cold reaction was poured onto crushed ice, the solid obtained was filtered, washed with water, dried and recrystallized from ethanol.

3-Allyl-2-thioxo-2, 3,5,6,7,8-hexahydro-1Hbenzo[4,5]thieno[2,3-d] pyrimidin-4-one(11)

M.p.: $234-237^{\circ} \mathrm{C}, \mathrm{IR}\left(\mathrm{cm}^{-1}\right) 3330,1710$, 1210; ${ }^{1} \mathrm{H}-N M R\left(\right.$ DMSO- $\left.\mathrm{d}_{6}\right)$ : 1.68-1.73, (m, $4 \mathrm{H}, 2 \mathrm{CH}_{2}$ at C-6 d C-7), 2.30-2.50(m, $2 \mathrm{H}, \mathrm{CH}_{2}$ at C-5), 2.892.90( $\mathrm{m}, 2 \mathrm{H}, \mathrm{CH}_{2}$ at C-8); 4.35-4.50(br. $\mathrm{m}, 2 \mathrm{H}, \mathrm{NCH}_{2}$ ), 5.41-5.60 (m, $\left.2 \mathrm{H},=\mathrm{CH}_{2}\right) ; 5.89-6.30(\mathrm{~m}, 1 \mathrm{H}, \mathrm{HC}=)$, $11.9(\mathrm{~s}, 1 \mathrm{H}, \mathrm{NH}) ; \mathrm{MS}: \mathrm{m} / \mathrm{z}(\%) ; 278(100)$ ( $\mathrm{C}_{13} \mathrm{H}_{14} \mathrm{~N}_{2} \mathrm{OS}_{2}$; CHN: Anal. Calcd.: C, 56.11; H: 5.03; $\mathrm{N}: 10.07$; Found: C: 56.60; H: 4.34; N: 9.85.

3-(Phenyl)-2-thioxo-5, 6,7,8-tetrahydro-1Hbenzo[4,5]thieno[2,3-d] pyrimidin-4-one(11 $)$ M.p.:261-263 ${ }^{\circ} \mathrm{C} ; \mathrm{IR}\left(\mathrm{cm}^{-1}\right): 3400,1700$, 1200; ${ }^{1} \mathrm{H}-\mathrm{NMR}\left(\mathrm{CDCl}_{3}\right): 1.80-1.84\left(\mathrm{~m}, 4 \mathrm{H}, 2 \mathrm{CH}_{2}\right.$ at $\mathrm{C}-6$ \& C-7), 2.58-2.61 (m, 2H, $\mathrm{CH}_{2}$ at C-5), 2.85$2.90(\mathrm{~m}, 2 \mathrm{H}, \mathrm{CH}$ at C-8), 7.30-7.60 (m, 5H, Ar- $\mathrm{H})$, 11.5(br.s, $1 \mathrm{H}, \mathrm{NH}$ ) MS: m/z (\%) $314\left[\mathrm{M}^{+}\right](100)$ $\mathrm{C}_{16} \mathrm{H}_{14} \mathrm{~N}_{2} \mathrm{OS}_{2} ; \mathrm{CHN}$ :Anal. Calcd, C: 61.14; $\mathrm{H}: 4.45$; $\mathrm{N}: 8.91$, Found: C: $61.20 ; \mathrm{H}: 4.53 ; \mathrm{N}: 8.90$.

3-(4-Chlorophenyl)-2-thioxo-5,6,7,8-tetrahydro$1 \mathrm{H}-$ benzo[4,5]thieno [2,3-d]pyrimidine-4-one (11)

M.p.: $289-291^{\circ} \mathrm{C}, \mathrm{IR}\left(\mathrm{cm}^{-1}\right) 3200,1700$, 1220; ${ }^{1} \mathrm{H}-\mathrm{NMR}\left(\mathrm{CDCl}_{3}\right): 1.70-1.76\left(\mathrm{~m}, 4 \mathrm{H}, \mathrm{CH}_{2}\right.$ at $\mathrm{C}_{6}$ \& $\left.\mathrm{C}_{7}\right)$ 2.60-2.64 (m, 2H, $\mathrm{CH}_{2}$ at $\left.\mathrm{C}-5\right)$; 2.71-2.74 (m, $2 \mathrm{H}, \mathrm{CH}_{2}$ at $\mathrm{C}_{8}$ ), 7.30-7.50 (m, $4 \mathrm{H}, \mathrm{Ar}-\mathrm{H}$ ); 8.5(br.s, $1 \mathrm{H}, \mathrm{NH}$ ); $\mathrm{MS}: \mathrm{m} / \mathrm{z}(\%) 348\left[\mathrm{M}^{+}\right](90) \mathrm{C}_{16} \mathrm{H}_{13} \mathrm{Cl}^{35} \mathrm{~N}_{2} \mathrm{OS}$, 350[M+2] (25) $\left(\mathrm{C}_{16} \mathrm{H}_{13} \mathrm{Cl}^{37} \mathrm{~N}_{2} \mathrm{OS}_{2}\right)$; CHN: Anal. Calcd (\%): C, 55.09; H,3.73; N, 8.03; Found: C, 55.30; H, 3.76; N, 8.09 .

General procedure for synthesis of : 3Substituted-2-hydrazino-5,6,7,8-tetrahydro-3Hbenzo[4,5]thieno[2,3-d]pyrimidin-4-ones.(12).

A mixture of compound $11_{\mathrm{b}}(1.0 \mathrm{mmol})$, and hydrazine hydrate $(99 \%)(2.0 \mathrm{mmol})$ in ethanol
(15 $\mathrm{ml}$ ) was submitted to microwave irradiation for $5 \mathrm{~min}$. at $(60 \mathrm{~W}) 100^{\circ} \mathrm{C}$. After cooling the reaction mixture poured into ice cooled water and stirred for $10 \mathrm{~min}$ the solid product was filtered, dried and recrystallized from ethanol.

3-Allyl-2-hydrazino-5,6,7,8-tetrahydro-3Hbenzo[4,5]thieno[2,3-d] pyrimidin-4-one (12 $\left.{ }_{\mathrm{a}}\right)$

M.p. $196-199^{\circ} \mathrm{C}$; IR $\left(\mathrm{cm}^{-1}\right): 3390,3310$, 1610, 1580, ${ }^{1} \mathrm{H}-\mathrm{NMR}: 1.70-1.73\left(\mathrm{~m}, 4 \mathrm{H}, 2 \mathrm{CH}_{2}\right.$ at C$6 \mathrm{~d} \mathrm{C}-7) ; 2.61-2.68\left(\mathrm{~m}, 2 \mathrm{H}, \mathrm{CH}_{2}\right.$ at C-5); $2.80-2.82(\mathrm{~m}$, $2 \mathrm{H}, \mathrm{CH}_{2}$ at $\mathrm{C}-8$ ), 4.01-4.30 (br. s, $2 \mathrm{H}, \mathrm{NH}_{2}$ ), 4.50$4.56\left(\mathrm{~m}, 2 \mathrm{H}, \mathrm{NCH}_{2}\right), 4.96\left(\mathrm{~d} \mathrm{~d}, 1 \mathrm{H}, \mathrm{J}=10.5 \mathrm{~Hz},=\mathrm{CH}_{2}\right)$, 5.12, (dd, $\left.1 \mathrm{H}, \mathrm{J}=17.5 \mathrm{~Hz},=\mathrm{CH}_{2}\right), 5.80-5.84(\mathrm{~m}, 1 \mathrm{H}$, $\mathrm{CH}=) ; 8.5$ (br.s, $1 \mathrm{H}, \mathrm{NH}) \cdot \mathrm{MS}: \mathrm{m} / \mathrm{z}(\%) 276\left[\mathrm{M}^{+}\right](90 \%)$,( $\mathrm{C}_{13} \mathrm{H}_{16} \mathrm{~N}_{4} \mathrm{OS}$ ); CHN Anal. Calcd.\%: C, 56.52; H, 5. 79; N, 20.28; Found :C,56.56; H, 5.83; N,20.24.

3-(4-Chlorophenyl)-2-hydrazino-5, 6,7,8tetrahydro-3H-benzo[4,5]-thieno[2,3-d]pyrimidin4-one $\left(12_{b}\right)$

M.p.204-206 C; IR( $\left.\mathrm{cm}^{-1}\right), 3410$ (br.), 1610, 1580 ; ${ }^{1} \mathrm{H}-\mathrm{NMR}$ (DMSO-d $\mathrm{d}_{6}$ ) 1.74-1.80(m, 4H, $2 \mathrm{CH}_{2}$ at C-6 d C-7); 2.63-2.70( $\mathrm{m}, 2 \mathrm{H}, \mathrm{CH}_{2}$ at C-5), 2.80$2.83\left(\mathrm{~m}, 2 \mathrm{H}, \mathrm{CH}_{2}\right.$ at $\left.\mathrm{C}-8\right), 4.3$ (br. s, $2 \mathrm{H}, \mathrm{NH}_{2}$ ), 7.47.9 (m, 4H, Ar-H), 8.01 (br. s, $1 \mathrm{H}, \mathrm{NH})$; MS: $\mathrm{m} / \mathrm{z}(\%)$ $346\left[\mathrm{M}^{+}\right]$(90) $\left(\mathrm{C}_{16} \mathrm{H}_{15}{ }^{35} \mathrm{Cl} \mathrm{N}{ }_{4} \mathrm{OS}\right), 348[\mathrm{M}+2]$ (25) $\left(\mathrm{C}_{16} \mathrm{H}_{15}{ }^{37} \mathrm{ClH}_{4} \mathrm{OS}\right) ; \mathrm{CHN}$ : Anal. Calcd: C,55.49; $\mathrm{H}$, 4.33; N , 16.18 ; Found: C, 55.45; H: 4.30; N: 16.14.

4-Allyl-6,7,8,9-tetrahydro-4H-benzo[4,5] thieno [2,3-d] $[1,2,4]$ triazolo $[3,4,-$ b]pyrimidin-5-one (13a)

A mixture of compound $12_{\mathrm{a}}(1.0 \mathrm{mmol})$ and formic acid $(10 \mathrm{ml})$ was submitted to microwave irradiation for $10 \mathrm{~min}$. at $(60 \mathrm{~W}) 80^{\circ} \mathrm{C}$ after cooling the reaction mixture was poured into ice-cooled water, the formed solid was collected by filtration washed several time with ethanol, dried and recrystallized from ethanol.: M.p. $213-215^{\circ} \mathrm{C}, \mathrm{IR}\left(\mathrm{cm}^{-}\right.$ $\left.{ }^{1}\right), 1680,1610,{ }^{1} \mathrm{H}-\mathrm{NMR}\left(\mathrm{CDCl}_{3}\right): 1.80-1.86(\mathrm{~m}, 4 \mathrm{H}$, $2 \mathrm{CH}_{2}$ at $\mathrm{C}_{7}$ and $\mathrm{C}-8$ ), 2.60-2.78( $\mathrm{m}, \mathrm{CH}_{2}$ at $\mathrm{C}-6$ ), 3.00-3.42 (m, $2 \mathrm{H}, \mathrm{CH}_{2}$ at $\left.\mathrm{C}-9\right)$; $4.50-4.56(\mathrm{~m}, 2 \mathrm{H}$, $\mathrm{NCH}_{2}$ ), 4.96(dd, $\left.1 \mathrm{H}, \mathrm{J}=10.5 \mathrm{~Hz},=\mathrm{CH}_{2}\right), 5.12$, (dd, $\left.1 \mathrm{H}, \mathrm{J}=17.5 \mathrm{~Hz},=\mathrm{CH}_{2}\right), 5.80-5.84(\mathrm{~m}, 1 \mathrm{H}, \mathrm{C}-\mathrm{H}=) ; 8.5$ (br. s, $1 \mathrm{H}, \mathrm{NH}) ; 9.30(\mathrm{~s}, 1 \mathrm{H}$, triazolo $-\mathrm{H})$; $\mathrm{MS}, \mathrm{m} / \mathrm{z}=$ $286(90)\left(\mathrm{C}_{14} \mathrm{H}_{14} \mathrm{~N}_{4} \mathrm{OS}\right)$; $\mathrm{CHN}$ : Anal. Calcd :C, 58.74 ;H, 4.89; N: 19.58. Found: C: 58.79; H: 4.80; N; 19.55. 
General procedure for synthesis of: 4Substituted-1-methyl-6,7,8,9-tetrahydro-4Hbenzo[4,5]thieno[2,3-d][1,2,4]triazolo[3,4-b] Pyrimidine 5-ones $\left(13_{b, c}\right)$

A mixture of compound $12_{a, b}(1.0 \mathrm{mmol})$ and acetic acid $(10 \mathrm{ml})$ was submitted to microwave irradiation for $10 \mathrm{~min}$ at $(60 \mathrm{~W}) 80^{\circ} \mathrm{C}$. After cooling the reaction mixture was poured into ice-cooled water. The solid product was collected by filtration washed several time with ethanol, dried and recrystallized from ethanol.

4-Allyl-1-methyl-6,7,8,9-tetrahydro-4H-benzo $[4,5]$ thieno[2,3-d][1,2,4] triazolo [3,4-b] pyrimidin5-one $\left(13_{b}\right)$

M.p.: $228-230^{\circ} \mathrm{C}$; IR $\left(\mathrm{cm}^{-1}\right): 1700,1610 ;{ }^{1} \mathrm{H}-$ NMR (DMSO- $\left.\mathrm{d}_{6}\right): 1.78-1.89\left(\mathrm{~m}, 4 \mathrm{H}, 2 \mathrm{CH}_{2}\right.$ at C-7\& $\mathrm{C}-8), 2.6\left(\mathrm{~m}, 2 \mathrm{H}, \mathrm{CH}_{2}\right.$ at C-6) ,2.76 (s, 3H, $\left.\mathrm{CH}_{3}\right)$, 2.96$3.2(\mathrm{~m}, 2 \mathrm{H}$ at C-9); 4.57-4.70 (m, 2H, N-CH $), 5.10$ (dd, $\left.1 \mathrm{H}, \mathrm{J}=19.8, \mathrm{CH}_{2}\right), 5.40\left(\mathrm{dd}, \mathrm{H}, \mathrm{J}=17.5, \mathrm{CH}_{2}=\right)$ 6.03(m, $1 \mathrm{H}, \mathrm{HC}=)$; MS: m/z $\left[\mathrm{M}^{+}\right] 300$ (100); $\mathrm{C}_{15} \mathrm{H}_{16} \mathrm{~N}_{4}$ OS. CHN : Anal. Calcd: C,60.00; H, 5.33; N, 18.66, Found: C,60.50; H, 5.40; N,18.65.

4-(4-Chlorophenyl)-1-methyl-6,7,8,9-tetrahydro4H-benzo[4,5] thieno[2,3-d][1,2,4] triazolo[3, 4,-b] pyrimidin-5 -one (13)

M.p.: $300<$, IR $\left(\mathrm{cm}^{-1}\right): 1680,1610 ;{ }^{1} \mathrm{H}-\mathrm{NMR}$ (DMSO- $\mathrm{d}_{6}$ ): 1.80-1.86 (m, $4 \mathrm{H}, 2 \mathrm{CH}_{2}$ at C-7 d C-8) $2.5-268\left(\mathrm{~m}, 2 \mathrm{H}, \mathrm{CH}_{2}\right.$ at $\left.\mathrm{C}-6\right) ; 2.73\left(\mathrm{~s}, 3 \mathrm{H}, \mathrm{CH}_{3}\right)$, 3.00-3.20 (m, 2H, $\mathrm{CH}_{2}$ at $\left.\mathrm{C}-9\right)$ 7.30-7.70 (m, 4H, Ar-H); MS: m/z(\%) $370\left[\mathrm{M}^{+}\right](100)\left(\mathrm{C}_{18} \mathrm{H}_{15}{ }^{35} \mathrm{CIN}_{4} \mathrm{OS}\right.$; 372[M+2] (40) $\left(\mathrm{C}_{18} \mathrm{H}_{15}{ }^{37} \mathrm{CIN}_{4} \mathrm{OS}\right)$; CHN: Anal. Calcd. C: 58.29; H: 4.04; N: 15.11. Found :C, 58.62; H, 4.09; N, 15.12.

General procedure for synthesis of: 4Substituted-1-mercapto-6,7,8,9-tetrahydro-4Hbenzo[4,5]thieno[2,3-d][1,2,4]triazolo[3,4b]pyrimidin-5-ones $\left(14_{\mathrm{a}, \mathrm{b}}\right)$.

A mixture of $12_{\mathrm{a}, \mathrm{b}} \quad(1.0 \mathrm{mmol})$ and carbon disulphide $(10 \mathrm{~mL})$ in pyridine $(10 \mathrm{~mL})$ was stirred for 10 min., then submitted to microwave irradiation for $10 \mathrm{~min}$ at $(60 \mathrm{~W}) 40^{\circ} \mathrm{C}$. The mixture was evaporated under reduced pressure and the residue was treated with ethanol. The formed solid was filtered and washed several times with water, dried, and recrystallized from ethanol.
4-Allyl-1-mercapto-6,7,8,9- tetrahydrobenzo[b] thieno[2,3-d][1,2,4] triazolo[3,4-b] pyrimidin $5(4 \mathrm{H})$ one (14a)

m.p. : $246-248{ }^{\circ} \mathrm{C}$, IR $\left(\mathrm{Cm}^{-1}\right): 3200,1680$, 1210; ${ }^{1} \mathrm{H}-\mathrm{NMR}\left(\mathrm{CDCl}_{3}\right) 1.78-1.81\left(\mathrm{~m}, 4 \mathrm{H}, \mathrm{CH}_{2}\right.$ at C$7 \mathrm{~d} \mathrm{C}-8), 2.65-2.71\left(\mathrm{~m}, 2 \mathrm{H}, \mathrm{CH}_{2}\right.$ at $\left.\mathrm{C}-6\right) ; 2.95-3.00$ (m, $2 \mathrm{H}, \mathrm{CH}_{2}$ at C-9); 4.48-4.60 (m, 2H, N-CH $), 5.00$ (dd, $\left.1 \mathrm{H}, \mathrm{J}=9.8 \mathrm{~Hz},=\mathrm{CH}_{2}\right) ; 5.27(\mathrm{dd}, 1 \mathrm{H}, \mathrm{J}=16.8 \mathrm{~Hz}$, $\left.=\mathrm{CH}_{2}\right)$ 5.86-5.91 (m, $\left.1 \mathrm{H}, \mathrm{HC}=\right)$; 10.6 (br. s, $\left.1 \mathrm{H}, \mathrm{SH}\right)$; MS: $\mathrm{m} / \mathrm{z}(\%),\left[\mathrm{M}^{+}\right] 318(100)\left(\mathrm{C}_{14} \mathrm{H}_{14} \mathrm{~N}_{4} \mathrm{OS}_{2}\right.$; $\mathrm{CHN}$ : Anal. Cacld. (\%): C, 52.83; H, 4.40; N, 17.61. Found C: $52.89 ; \mathrm{H}: 4.48 ; \mathrm{N}: 17.69$.

4-(Chlorophenyl)-1-mercapto-6,7,8,9tetrahydrobenzo[b]thieno[2,3-b] $[1,2,4]$ triazolo [3,4-b] pyrimidin-5 (4H)-one (14 $)$

M.p.: $260-263^{\circ} \mathrm{C}$, IR $\left(\mathrm{cm}^{-1}\right): 3300,1600$, 1610, 1200; ${ }^{1} \mathrm{H}-\mathrm{NMR}\left(\mathrm{CDCl}_{3}\right): 1.80-1.84(\mathrm{~m}, 4 \mathrm{H}$, $2 \mathrm{CH}_{2}$ at $\left.\mathrm{C}-7 \mathrm{~d} \mathrm{C}-8\right) ; 2.50-2.62\left(\mathrm{~m}, 2 \mathrm{H}, \mathrm{CH}_{2}\right.$ at $\left.\mathrm{C}-6\right)$; 2.95-3.2( $\mathrm{m}, 2 \mathrm{H}, \mathrm{CH}_{2}$ at C-9), 7.1-7.80 (m, 4H, Ar-H), 11.5 (br. s, 1H, SH); MS: m/z(\%) ,[M+] 388 (90) $\left(\mathrm{C}_{17} \mathrm{H}_{13}{ }^{35} \mathrm{CIN}_{4} \mathrm{OS}_{2}\right), 390[\mathrm{M}+2](25)\left(\mathrm{C}_{17} \mathrm{H}_{13}{ }^{37} \mathrm{CIN}_{4} \mathrm{OS}_{2}\right)$; CHN Anal. Calcd. (\%) : C, 52.50; H, 3.34; N, 14.41. Found: C, 52.59; H, 3.40; N, 14.40.

\section{RESULTS AND DISCUSSION}

The starting materials, namely 2-amino-3(carboxyamide or carboxylic acid)-4,5-disubtituted thiophene $1_{a, b}$ were prepared according to the corresponding literature methods ${ }^{18,19}$.

The reaction of $1_{a, b}$ and formamide under a microwave irradiation with reactants ratio of $1: 2$ at $1: 5-6$ in the presence of one equivalent of acetic acid at $(300 \mathrm{~W})$ for $10 \mathrm{~min} 130^{\circ} \mathrm{C}$ yielded thienopyrimidinone derivatives $4_{\mathrm{a}-\mathrm{c}}$ (Scheme 1, Table 1).

The next step we converted $3 \mathrm{H}$ thieno[2,3-d]pyrimidin-4-one $4_{b}$ in to 4chlorothieno[2,3-d]pyrimidine derivatives 7 by the action of phosphorous oxychloride using both classical and microwave reaction ${ }^{(11,12,20-22)}$; the latter method afforded higher yields of the required products in shorter time.(Scheme 3, Table 2)

Substitution with an aromatic amine was performed on chloro derivatives under classical 
and microwave irradiation to produce the anilino derivatives $8_{\text {a-c }}$ (Scheme 3, Table 2). The structure of synthesized compounds were assigned on the basis of its spectral data and elemental analysis. Next attention was focused on the prep. In the classical synthesis of this compounds, a mixture of starting material reacted with alkyl or aryl thio isocyanates in ethanol. This method provided low to moderate yield and long reaction time ${ }^{(22)}$. We have recently synthesis the target compounds $\mathbf{1 1}_{\mathrm{a}-\mathrm{c}}$ using microwave irradiation in acetic acid (Scheme 4, Table 3).

Table 1: Microwave experimental condition for the synthesis of compound 4:

\begin{tabular}{llllllllll}
\hline $\begin{array}{l}\text { Compound } \\
\text { No. }\end{array}$ & $\mathbf{R}_{1}$ & $\mathbf{R}_{2}$ & $\mathbf{X}$ & $\begin{array}{l}\text { Substrate } \\
\text { ratio }\end{array}$ & $\begin{array}{l}\text { Temp. } \\
\left({ }^{\circ} \mathrm{C}\right)\end{array}$ & $\begin{array}{l}\text { Power in } \\
\text { put }(\mathbf{W})\end{array}$ & $\begin{array}{l}\text { Yield } \\
(\%)\end{array}$ & $\begin{array}{l}\text { M.p. } \\
\left({ }^{\circ} \mathbf{C}\right)\end{array}$ & $\begin{array}{l}\text { Lit..m. } \mathbf{p}^{24} \\
\left({ }^{\circ} \mathbf{C}\right)\end{array}$ \\
\hline $1_{a}$ & $\mathrm{CH}_{3}$ & $\mathrm{CH}_{3}$ & $\mathrm{NH}_{2}$ & $1: 6$ & 130 & 300 & 85 & $180-183$ & \\
$1_{b}$ & $\left(\mathrm{CH}_{2}\right)_{4}$ & & $\mathrm{NH}_{2}$ & $1: 5$ & 130 & 300 & 86 & $257-259$ & $255-275$ \\
$1_{c}$ & $\left(\mathrm{CH}_{2}\right)_{5}$ & & $\mathrm{NH}_{2}$ & $1: 5$ & 130 & 300 & 81 & $210-211$ & $209-211$ \\
$1_{a}$ & $\mathrm{CH}_{3}$ & $\mathrm{CH}_{3}$ & $\mathrm{OH}$ & $1: 6$ & 130 & 300 & 79 & $180-183$ & - \\
$1_{b}$ & $\left(\mathrm{CH}_{2}\right)_{4}$ & & $\mathrm{OH}$ & $1: 5$ & 130 & 300 & 83 & $257-259$ & $255-275$ \\
$1_{c}$ & $\left(\mathrm{CH}_{2}\right)_{5}$ & & $\mathrm{OH}$ & $1: 5$ & 130 & 300 & 81 & $210-211$ & $209-211$ \\
\hline
\end{tabular}

Table 2: Experimental conditions used for the microwave assisted and classical methods synthesis of compounds $7 \& 8$

\begin{tabular}{lccccccc}
\hline $\begin{array}{l}\text { Compound. } \\
\text { No. }\end{array}$ & Ar & $\begin{array}{c}\text { Microwave method } \\
\text { Reaction } \\
\text { time (min.) }\end{array}$ & Yield \% & $\begin{array}{c}\text { Classical Method } \\
\text { Reaction Yield \% } \\
\text { time (h.) }\end{array}$ & $\begin{array}{c}\text { M.p. } \\
\left({ }^{\circ} \mathrm{C}\right)\end{array}$ & $\begin{array}{c}\text { Lit..m.p. }^{24} \\
\left({ }^{\circ} \mathbf{C}\right)\end{array}$ \\
\hline 7 & - & 15 & 75 & 20 & 48 & $93-95$ & $90-92$ \\
$8_{a}$ & $4-\mathrm{CH}_{3}-\mathrm{C}_{6} \mathrm{H}_{4}$ & 20 & 85 & 12 & 13 & $235-237$ & - \\
$8_{b}$ & $3-\mathrm{CF}_{3} \mathrm{C}_{6} \mathrm{H}_{4}$ & 20 & 76 & 12 & 12 & $224-225$ & $224-226$ \\
8 & $2,5-\mathrm{CH}_{3} \mathrm{C}_{6} \mathrm{H}_{4}$ & 20 & 84 & 12 & 15 & $213-215$ & - \\
\hline
\end{tabular}

a; Reaction conditions: 2-PrOH, reflux under MW irradiation (60 W)

b: Reaction conditions: 2-PrOH, reflux temperature.

Table 3: Microwave experimental conditions for the synthesis of compounds 11-14:

\begin{tabular}{|c|c|c|c|c|c|c|c|}
\hline $\begin{array}{l}\text { Comp. } \\
\text { No. }\end{array}$ & $\mathbf{R}$ & $\begin{array}{c}\text { Temp. } \\
\left({ }^{\circ} \mathrm{C}\right)\end{array}$ & $\begin{array}{c}\text { Power in } \\
\text { put (W) }\end{array}$ & $\begin{array}{c}\text { Time } \\
\text { (min.) }\end{array}$ & $\begin{array}{c}\text { Yield } \\
(\%)\end{array}$ & $\begin{array}{l}\text { M.P. } \\
\left({ }^{\circ} \mathrm{C}\right)\end{array}$ & $\begin{array}{l}\text { Lit.m.p }{ }^{24} \\
\quad\left({ }^{\circ} \mathrm{C}\right)\end{array}$ \\
\hline $11_{\mathrm{a}}$ & $\mathrm{CH}_{2} \mathrm{CH}=\mathrm{CH}_{2}$ & 120 & 300 & 20 & 85 & $234-235$ & - \\
\hline $11_{b}$ & $\mathrm{C}_{6} \mathrm{H}_{5}$ & 120 & 300 & 20 & 87 & $261-263$ & $259-261$ \\
\hline $11_{c}$ & $4-\mathrm{ClC}_{6} \mathrm{H}_{4}$ & 120 & 300 & 20 & 85 & $289-291$ & $289-290$ \\
\hline $12_{a}$ & $\mathrm{CH}_{2} \mathrm{CH}=\mathrm{CH}_{2}$ & 100 & 60 & 5 & 80 & $196-198$ & - \\
\hline $12_{b}$ & $4-\mathrm{ClC}_{6} \mathrm{H}_{4}$ & 100 & 60 & 5 & 86 & 204-206 & 204-204 \\
\hline $13_{a}$ & $\mathrm{CH}_{2} \mathrm{CH}=\mathrm{CH}_{2}$ & 80 & 60 & 10 & 87 & 213-215 & - \\
\hline $13_{b}$ & $\mathrm{CH}_{2} \mathrm{CH}=\mathrm{CH}_{2}$ & 80 & 60 & 10 & 83 & $228-230$ & - \\
\hline $13_{c}$ & 4- $\mathrm{ClC}_{6} \mathrm{H}_{4}$ & 80 & 60 & 10 & 85 & $>300$ & 300 \\
\hline $14_{a}$ & $\mathrm{CH}_{2} \mathrm{CH}=\mathrm{CH}_{2}$ & 40 & 60 & 10 & 83 & $246-248$ & - \\
\hline $14_{b}$ & $4-\mathrm{ClC}_{6} \mathrm{H}_{4}$ & 40 & 60 & 10 & 87 & $260-263$ & - \\
\hline
\end{tabular}


The MW method requires higher yields, short reaction time, and eco friendly. Reaction of $11_{\text {a-c }}$ with hydrazine hydrate under microwave irradiation at $60 \mathrm{~W}\left(100^{\circ} \mathrm{C}\right)$ for $5 \mathrm{~min}$., afforded 2hydrazino derivatives $12_{a, b}$. (Scheme 4, Table 3). Cyclization of compound $12_{\mathrm{a}, \mathrm{b}}$ by aliphatic carboxylic acid, namely Formic or acetic acid under $\mathrm{MW}$ irradiation for $10 \mathrm{~min}$ at $(60 \mathrm{~W}) 80^{\circ} \mathrm{C}$ gave the triazolo derivatives $13_{\mathrm{a}}$ and $13_{\mathrm{b}, \mathrm{c}}$ respectively (Scheme 4,Table 3).

Finally, treatment of $12_{a, b}$ with carbon disulphide under microwave irradiation for $10 \mathrm{~min}$. yielded mercaptotriazolo derivatives $14_{\mathrm{a}, \mathrm{b}}$ (Scheme 4, Table 3).

The structure of compounds 11-14 were confirmed from their spectral data and elemental analysis.

\section{Antimicrobial activity}

The antimicrobial activity of the compounds considered was tested on the following organisms:

$1 \quad$ Candida albicans ATCC No. 10231

2 Staphylocoecus Aureus ATCC No. 6538.

3 Eschierichia Coli ATCC No. 8739.

4 Proteus mirabilis ATCC No. 29906.

The biological activity was planned according to the cup-plate method adopted with some modify ${ }^{23}$

What man No. 2 filter paper disk $(6.5 \mathrm{~cm})$ was impregnated with $200 \mathrm{mg}$ of the compound. The disk was placed on the surface of the cold solid medium petridishes vaccinated with considered organisms and then incubated at $5^{\circ} \mathrm{C}$ for $1 \mathrm{~h}$. to permit good diffusion and then transferred to an incubated at $28^{\circ} \mathrm{C}$ for $24 \mathrm{~h}$.

A summary of the biological activity results is shown in Table 4.

Table 4: Antimicrobial activity of the compounds against bacteria and fungi

\begin{tabular}{lcccccccc}
\hline \multirow{2}{*}{ Test } & \multicolumn{7}{c}{ Compounds } \\
\cline { 2 - 8 } Organism & 5 & 7 & $\mathbf{8}_{\mathrm{b}}$ & $11_{\mathrm{a}}$ & $12_{\mathrm{b}}$ & $13_{\mathrm{a}}$ & $\mathbf{1 3}_{\mathrm{b}}$ & $1_{\mathrm{a}}$ \\
\hline 1 & + & + & - & + & - & - & + & - \\
2 & + & ++ & + & - & - & - & + & + \\
3 & + & + & - & - & - & - & + & + \\
4 & - & - & - & + & + & - & - & - \\
\hline
\end{tabular}

The sensitivity of microorganisms to the compound is identified in the following manner:

$+++=$ highly sensitive (inhibition zone $1.2-1.5 \mathrm{~mm}$ )

$++=$ fairly sensitive (inhibition zone 1.2-0.9 mm)

$+=$ Slightly sensitive (inhibition zone 0.9-0.6 mm)

$=$ not sensitive.

\section{REFERENCES}

1. Litvinov, V. P.,Russian Chemical Bulletin 2004, 53(3), 487--516.

2. Alagarsmy, V.; Murngarathan, G.; Venkateshperumal, R.;Biol. Pharm. Bull .2003, 26(12), 1711-1714.

3. Vikunjana K. A.;Pragnesh D. ; Keshav C. P., International Journal of Chem. Tech. research2013,5(1), 142-155.

4. Gopalsamy, A.; Yang, H.; Ellingboe, J.W.; Tsou, H.R; Zhang, N.; Honores, E.; Powell, D., Miranda, M.; McGinnis, J.P; Robindran, S.k., Bio. Org. Med. Chem.2005, 15(6), 1591-1594.

5. Baselga, J.; Averbuch, S. D., Drugs2000,60(1), 33-40. 
6. Habib, N.S.; Soliman,R.; El-Tombary, A.;. ElHawash, S. A ;Shaaban O. G.,Medicinal Chem. Research 2013,22(7), 3289-3308.

7. Dang,L.;Gui,H.;Zhou,Q.,Wuhan University J. of Natural Science 2012, 17(2), 177-184.

8. El-Azab, A. A.;Al-Omar, M. A.;Abdel-Aziz ,A .A- M.;Abdel-Aziz, N. I. ;El-Sayed, M. A. A.;Aleisa, A.M.;Sayed-Ahmed ,M.M.;AbdelHamide, S. G.,European Journal of Medicinal Chemistry2010,45(9), 4188-4198.

9. Veena, K.; Nurgund, L.S.;Nurgund, L.S; Chandra, J. N. N.;Nurgund,L. V.,Derpharma Chemica 2012, 4(2), 581-586.

10. Chay kovsky, M.;Lin, M.;Rosowsky, A.; Modest, E.,J. Med. Chem.1977, 16, 188-191.

11. Li, F.; Wang, Q.; Ding, Z.; Tao, F., Org. Lett. 2003, 5, 2169-2171.

12. Besson, T.; Dozias, M.J.; Guillard, J.; Jacquault, P.;Legoy, M.D.; Rees, C.W., Tetrahedron 1998,54, 6475-6484.

13. Akhil, A. N.; Ashish, p.; Rajesh, K. S.; Kishore, R. D.;Rathi, L. G.; Pharmagene2013, 1, 16.[www.genesisjournals.org]

14. Mehta,S.; Swarnkar,N.; Vyas, M.; Vardia, J.; Panjabi,P. B. ;Ameta, S.C.,Bull. Korean Chem. Soc.2007,28(12), 2338-2342.

15. Mizuno, T.; Iwai, T.; Ishino Y., Tetrahedron
Lett.2004, 45, , 7073-7075.

16. Alexandre,F.R.; Berecibar, A.;Wrigglesworth, R.;Besson, T., Tetrahedron Lett. 2003, 44, , 4455-4458.

17. Hesse, J.; Perspicace,E.; Kirch, G., Tetrahedron Lett. 2007, 48, 5261-5264.

18. Sabins, R. W.; Rangnekar, D. W.; Sonawane N. D., J. heterocyclic Chem. 1999, 36, , 333343.

19. Gewald,K.; Schinike, E.,Chem. Ber. 1966,99, 94-100; Chem. Abstr. 1976, 64, 8118c.

20. Alexandre, F. R. ;Berecibar A.;Besson T., Tetrahedron Lett. 2002, 43, 3911-3913.

21. Li, F.; Feng, Y.; Meng, Q.; Li, M.; Li Z.; Wang, G. ; Tao, F. ,ARKIVOC2007,(i) , 40-50.

22. Liu, G.; Song, B.; Yang, S.; Yue, W., Molecules 2006,11, 272-278.

23. Besson,T.;Chosson,E., Combinatorial Chemistry and High Throughput Screening 2007, 10, 1903-917.

24. El-Baih, F. E.; Al-Blowy, H. A. S. ; Al-Hazimi, M.,Molecule 2006, 11, 498-513.

25. El-Hiti, G. A.; Hussain A.; Hegazy, A. S. ; Alotaibi, M. H., J. of Sulfur Chemistry 2011,32(4), 361-395.

26. Abau-Zeid, A. A. ; Shehata, Y. M.,Indian J. Pharmacy 1969, 31, 72-75. 